\title{
Measuring Size of an Object using Computer Vision
}

\section{T. Dhikhi, Allagada Naga Suhas, Gosula Ramakanth Reddy, Kanadam Chandu Vardhan}

\begin{abstract}
In our research paper, the original size of an object present in an image is calculated. The choosed algorithms are used computer vision are carried out, it includes basic tools that are efficient enough to find the size of an object. In this project, we proposed the measuring of dimensions of an objects present in the picture and the distances between the objects is computed. Computing the measurements of the objects in a picture is similar to finding the distance to an object from the camera. First of all, we have to find the value of pixels per a given metric. To find this value, we have to select one reference object from the image by considering two major properties. First property is that dimensions (width \& height) of the reference object should be known and in terms of (inches or $\mathrm{mm}$ ) and the other property is that identification of the reference object should be easy.
\end{abstract}

Keywords-camera; image; computer vision; object size; size estimation.

\section{INTRODUCTION}

There are few methods to determine this, The most familiar that you probably noticed would be in crime scene photos when an object of known size such as a pencil or even a ruler is placed next to the object of interests. From this it is very easy to estimate the size of the object of interest.

If the object distance \& the focal length are known then we can able to compute the original measurements of an object present in a picture. The lens view fieldwith degrees as unit will be given by focal length.

Assume a sample, that a $10 \mathrm{~cm}$ lens with a vertical view field of 15 degrees on a camera by full frame. Therefore that occupies the half of the frame and uses field view of 8 degrees.

In this project, themeasuring of dimensions of an objects in the picture and the distances between the object is computed. Computing the measurements of the objects in a picture is similar to finding the distance to an object from the camera. First of all, we have to find the value of pixels per a given metric.To find this value, we have to select one reference object from the image by considering two major properties

Property-1:-The dimensions(width \& height) of the reference object should be known and in terms of (inches or $\mathrm{mm})$.

Property-2:- The identification of the reference object in a picture should be easy. The identification should be based on the object placement in a picture like placing the objects

Revised Manuscript Received on April 12, 2019.

T. Dhikhi, Assistant professor, CSE Department, SRMIST, Chennai, Tamilnadu. India.

Allagada Naga Suhas, CSE Department, SRMIST, Chennai, Tamilnadu.India(nagasuhas064@gmail.com)

GosulaRamakanth Reddy, CSE Department, SRMIST, Chennai, Tamilnadu. India (ramugosula99@gmail.com)

KanadamChanduVardhan, CSE Department, SRMIST, Chennai, Tamilnadu. India (chandu.vardhan007@gmail.com) at corners of the picture orbased on its appearances like its shape, color or any other factors. From the above cases, it is clear that our reference object in a picture should be identifiable uniquely compared to the other objects. These two properties are very important to find the measurements of an object present in a picture.

\section{FLOW DIAGRAM}

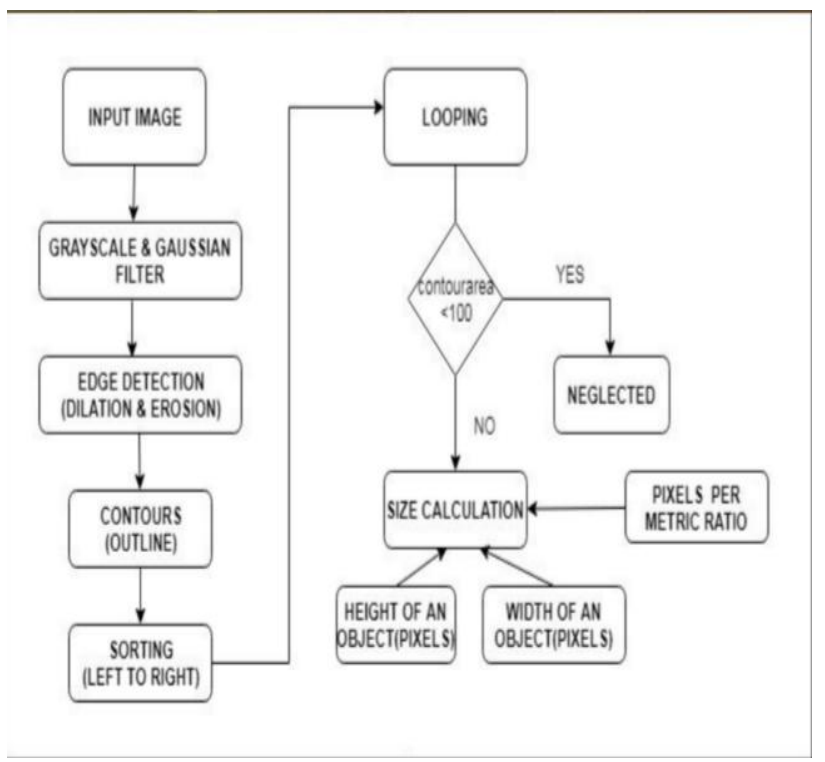

Fig.1.Flow diagram

As shown in the above fig.1., we have to store the input image in the memory. First give the path for reading the image present in the storage. Then perform the grayscale which identifies the objects present in the picture and also do gaussian filter to remove the unnecessary noise in the picture.

After filtering, the edge detection method is done to detect the edges of the object present in the picture. The outline is formed for the edge detected objects in the picture i.e., using contours. Then sort the objects present in the picture in sequential order.

If contour area is too small neglect the object otherwise calculate the dimensions of the object. To find the dimensions of the object we should know the pixels per metric ratio, Height \& Width (pixels). For finding pixels per metric ratio we need two parameters and they are Height $\&$ Width(inches) of the reference object. 


\section{WORKING CONCEPT}

\section{A.Requirements:}

The main aim is to find a solution for measuring the dimensions of an object present in a picture using algorithms related to computer vision. To make this possible, we have to consider two types of pictures - first type is with the presence of object and the second type without the presence of object in the picture. One more important factor is that to know the details about height of the camera.

We require prior idea on various libraries and frameworks, especifically CCV, BoofCV, CImg ,SimpleCV, OpenCV, Accord.Net. In Linux OS, Selected tool is available and it is an open-source software. In OpenCV, the algorithms for identification of real time objects, image conversion with various colour models to grayscale\& Harris corner detection etc to be found.

In this project work, the 3-Dimensional object is to be calculated is indicated by a 2-Dimensional area where the image background isinvisible. The $2 \mathrm{D}$ object representation being concave should not affect the output dimensions.

\section{B. Calculating the Width of the object:}

Camval is referred as the height from camera to image while capturing the picture and $\mathrm{x}$ is referred as the width parameter of an object (pixels). The $\mathrm{x}$ value indicates the operation given in the equ(1). By analysis ofdata, $y$ and $z$ values depends on the ratio camval parameter to the object's physical width. $y$ value can be acquired from the equ(2), $z$ value can be acquired from an equ(3).

$$
\begin{aligned}
& x=y^{*} K+z \\
& y=m_{1} * \stackrel{\text { camval }}{-b} \\
& \text { widRe al } \\
& z=m_{2} * \quad \frac{\text { camval }}{\text { widRe } a l}-b
\end{aligned}
$$

1

For finding the estimated width value we have to find the values of the equ((1),(2),(3)). P is referred as the estimated width.

$$
P=\square \square \text { camval } * \quad \frac{x+b_{1}}{m_{1} * K+b_{2}} \frac{* K+m_{2}}{*}
$$

\section{Calculating the Height of the object:}

Suppose consider a reference model camval value is as same as a theoretical model having the same height. Q is referred as the reference model and indicates the equ(5).

$$
Q=m_{1} * K+m_{2} * \text { camval }+b_{2}
$$

Herethe height parameter of the reference model can be obtained from equ(5) and indicated by Q. where $K$ is the parameter for values of distance in pixels of the lower border of displayed area and displayed object, camval is parameter that can be obtained from equ(1) and $b_{2}=203.7$, $m_{2}=0.06892, m_{1}=-1.028$ are the constant values that are acquired from the data analysis

$\mathrm{M}$ is referred as the the original height of the calculated object. The main goal is to acquire an estimated height parameter as equally close to the $M$ value. Distance between $\mathrm{real}_{0}$ and $\mathrm{real}_{2}$ is referred as reference model's physical height. The distance is the cam value, i.e., equal as the height parameter of the camera. $M$ is the parameter value indicates original object's displayed height(pixels) and $N$ refers to reference model's displayed height. In case of Logitech C920 HD Pro Webcam camera, gamma value is the twice the value of vertical angle of view.

The aim is to find estimated height value from the equ(6), that will be possibly equal to $M$ value. Camvalvalue is the height from camera to picture while capturing the pictures, with possible values of $\mathrm{K}$ coefficient is -1 or 1 . If $Q$, is greater than the pixHeig, then $\mathrm{K}$ value is -1 , otherwise $K$ value is 1 . Based on basic fundamental trigonometry rules, one can find the value of distance between real ${ }_{1}$ and real $_{2}$ from the equ(6) using basic trigonometry rules. The $\rho$ value can be to obtain from equ(7). y value is the substraction between angles values $\gamma$ and $\delta$, equ( $(8)$. $\delta$ value is possible to obtain from equ(9).

$$
\begin{aligned}
& \text { estHeig }=\text { camval }+k^{*} \tan (\rho) * D(6) \\
& \rho=\frac{\mid \text { pixHeig }-Q}{Q} * y \\
& y=\gamma-\delta \\
& \delta=\frac{K}{K+N} * \gamma
\end{aligned}
$$

$\mathrm{D}$ is referred as the camera's horizontal distance and the computed object, that is possible to obtain from the equ(10). The equ(10) uses the $\alpha$ values acquired from previously used equations.

$$
D=\cot (y)^{*} \text { camval }
$$

\section{E. The filtering of shadows and background:}

The object detection system provides two differentoptions for background and shadow filtering of the object. The primary option is finding the object measurements using RGB model; this solution from the object will be calculted with its shadow.

The another option is to remove the shadows formed from the object. The CIELab was selected for this work, after so many tests of different colormodels. By usingthe CIELab color model, it was found that it reduces the shadows formed by the objects in the photo.If the object is white, or in a shades of grey, or black, the CIELab model usage will be a drawback.

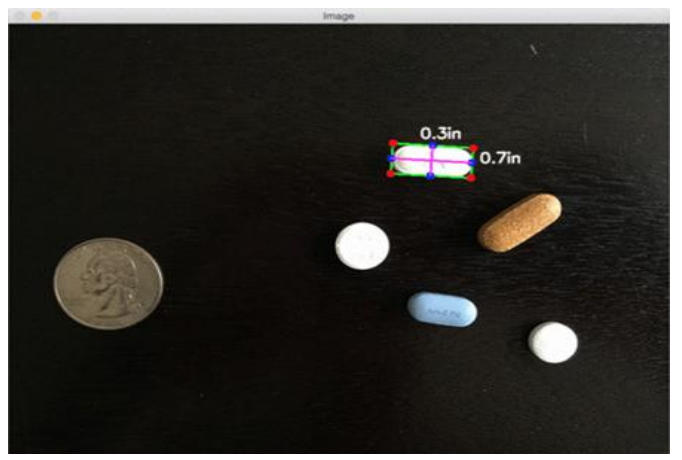

Fig.2. Sample picture

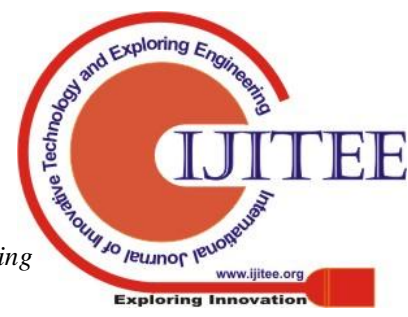


If the picture of an object is clicked in a situation where the shadows of the object will not affect the edge recognization in an object, so, the measurement of an object can be done without the need of shadows filtering. The detection is difficult when the object has a several areas with significantly various colors. In that type of cases, only any one of the areas with color is detected as the object.

\section{RESULTS \& DISCUSSIONS:}

The software application is assigned to shoot the image using camera with $44.4^{\circ}$ vertical angle of view. The results were examined with Logitech C920 HD pro Webcam camera, which satisfies the angle of view condition. The results of the application was obtained and noted by testing several number of images containing objects with various measurements, at various lengths from the camera and it is set to various heights. Values obtained from different tests are shown inTable.1.

\section{TABLE I.TestResults}

\begin{tabular}{|c|c|c|c|} 
& $\begin{array}{c}\text { original } \\
\text { measurements } \\
\text { of an object }\end{array}$ & yPos & size \\
{$[\mathrm{mm}]$} & $\mathrm{w} \times \mathrm{h}$ & $\mathrm{w} \times \mathrm{h}$ \\
& {$\left[\mathrm{mm}^{2}\right]$} & & {$\left[\mathrm{mm}^{2}\right]$} \\
\hline 153 & $96 \times 152$ & 59 & $92 \times 154$ \\
\hline 152 & $53 \times 81$ & 3 & $53 \times 87$ \\
\hline 127 & $46 \times 142$ & 71 & $52 \times 153$ \\
\hline 81 & $155 \times 95$ & 21 & $148 \times 101$ \\
\hline 352 & $72 \times 130$ & 103 & $80 \times 132$ \\
\hline 276 & $42 \times 141$ & 3 & $43 \times 128$ \\
\hline 142 & $139 \times 52$ & 44 & $146 \times 145$ \\
\hline
\end{tabular}

By observing the above table, cam value represents the height from where the camera shootsimages of an object, the original measurments of an object is a true dimensions of the object $\left[\begin{array}{lll}\mathrm{w} & \mathrm{x} & \mathrm{h}\end{array}\right], \mathrm{yPos}$ represents lower vertical coordinates of an object present in the image and the Size is the application output for the test. The test results obtainedis recorded as[w x h]. Thus, Tests conducted were successful and the measurements obtained has a variation of $7-10 \%$ when compared with original size of an image.

\section{CONCLUSION}

In thisresearch paper, a method for finding the object measurements present in an images using a camera was explained and related execution of software is explained. The identification of an object is based on taking two images in the same scenario without and with the object present in an image. The identified objects is distinguished by the size of object area displayed and the co-ordinates. The output obtained from these images are taken as input data for the equations to calculate the size. Based on the tests conducted, by considering various heights and distances of an object, andusing various objects, it was found that the deviation of the measurement from original measurement is less than $7-10 \%$.

\section{REFERENCES}

1. "Novel object-size measurements using digital camera"By LimengPu, RuiTian, Hsiao-Chun Wu, Kun Yan: IMCEC, 2016.

2. "Image-Based Distance and Area Measuring Systems" By Ming-Chih Lu, Wei-Yen Wang, \& Chun-Yen Chu: IEEE SENSORS JOURNAL, VOL. 6, NO. 2, APRIL 2006

3. "Principles of Digital Image Processing,"By M. J. Burge \& W. Burger,London, UK: Springer, 2009.

4. "Binary Digital Image Processing," By Y. M. Sharaiha\&S.Marchand-Maillet,London, UK: Academic Press, 1999.

5. "Single-Image Distance Measurement by A Smart Mobile Device" By Shangwen Chen, XianyongFang,JianbingShen\&Linbo Wang: IEEE Transactions on Cybernetics; September,2016 